\title{
Peritumoral lymphatic vessel density and invasion detected with immunohistochemical marker D240 is strongly associated with distant metastasis in breast carcinoma
}

\author{
Nur Fatiha Norhisham, Choi Yen Chong and Sabreena Safuan * (D)
}

\begin{abstract}
Background: Detection of vascular invasion by hematoxylin and eosin staining is the current pathological assessment practice to diagnose breast carcinoma. However, conventional hematoxylin and eosin staining failed to distinguish between blood vessel invasion and lymphatic vessel invasion. Both are important prognostic criteria however with different outcomes. The aim of this study is to distinguish between blood vessel invasion and lymphatic vessel invasion using conventional assessment and immunohistochemical markers. The prognostic significance of both circulatory invasions in invasive breast carcinoma was also investigated.

Methods: Consecutive sections of breast carcinoma samples from 58 patients were stained with CD34 and D240 to stain blood and lymphatic vessels respectively. Hematoxylin and eosin staining was carried out on another consecutive section as conventional staining.

Results: Although blood vessel density is higher in the sections (median $=10.3$ vessels) compared to lymphatic vessel density (median $=0.13$ ), vessel invasion is predominantly lymphatic invasion (69.8 and $55.2 \%$ respectively). Interestingly, peritumoral lymphatic vessel density and peritumoral lymphatic invasion was significantly associated with distant metastasis ( $p=0.049$ and $p=0.05$ respectively). The rate of false positive and false negative interpretation by hematoxylin and eosin was 46.7 and $53.3 \%$ respectively.
\end{abstract}

Conclusions: Lymphatic vessel invasion is a strong prognostic markers of breast carcinoma invasion and the use of immunohistochemical markers increase the rate and accuracy of detection.

Keywords: Lymphatic invasion, D2-40, CD34, Breast carcinoma

\section{Background}

Breast carcinoma is one of the leading cancer worldwide with more than 190,000 new cases reported each year in the US [1]. From this figure, the estimated deaths is about $21 \%$. The prognosis of breast cancer is based on the age and menopausal status of the patients, tumor size, histological types and grade, hormone and growth factor receptors and vascular invasion [2].

\footnotetext{
* Correspondence: sabreena@usm.my

School of Health Sciences, Universiti Sains Malaysia, 16150 Kubang Kerian, Kelantan, Malaysia
}

Currently, vascular invasion is detected microscopically as the presence of tumor cells within the blood or lymphatic vessels using haematoxylin and eosin $(\mathrm{H} \& \mathrm{E})$ staining [2]. Patients with positive vascular invasion often has poorer prognosis than those with negative invasion. The presence of vascular invasion was also associated with axillary lymph node involvement, systemic relapse and local recurrence [3, 4]. However, conventional assessment of vascular invasion by $H \& E$ stained slides was reported to have high rate of false interpretation. Retraction artefacts of the tissue sections tend to be reported as positive invasion while packed tumor cells in a vessel may be 
missed, leading to false positive and false negative observation of the tissue respectively. There was a low level of concordence (kappa $=0.3$ ) between two observers in reporting vascular invasion of $H \& E$ stained tissue section [5]. In addition, H\&E staining failed to distinguish between lymphatic vessel invasion and blood vessel invasion in patients' samples.

D240 is a monoclonal antibody that bind to an O-linked sialoglycoprotein on lymphatic endothelial cells but not on blood endothelial cells. Therefore, D240 antibody has been used as reliable marker to evaluate lymphatic invasion in many research settings $[6,7]$. The commercially available D240 antibody binds to a fixation resistant epitope on podoplanin molecules, an integral transmembrane glycoprotein. Ultrastructural analysis revealed the predominat localisation to the luminal surface of lymphatic vessels. CD34 is a common endothelial marker used to detect the presence of blood vessels. This antibody detects a $110 \mathrm{kDa}$ transmembrane glycoprotein expressed on endothelial cells, embryonic fibroblasts and some nervous tissues [8].

The aim of this study is to compare the incidence of lymphatic vessel invasion and blood vessel invasion in breast carcinoma cohort between conventional H\&E staining and immunohistochemical staining. The significance of using immunohistochemical markers in relation to adverse clinicopathological criteria is also evaluated.

\section{Methods}

\section{Patients and specimens}

This study was conducted on 58 consecutive formalin fixed paraffin embedded (FFPE) archival specimens of breast carcinoma obtained from Universiti Sains Malaysia Hospital. Clinical characteristics of the patients and tumors are summarized in Table 1.

\section{Immunohistochemistry}

Two consecutive FFPE breast carcinoma sections from each patient were stained with CD34 and D240 to assess blood vessels and lymphatic vessels respectively. Staining optimization was conducted on breast sections before using them in the main cohort. $4 \mu \mathrm{m}$ thick whole sections from each specimen were deparaffinized in two xylene baths for $5 \mathrm{~min}$ each and then rehydrated in a series of descending ethanol concentrations $(100,90,70$, 50 and $30 \%$ in water for $1 \mathrm{~min}$ at each concentration). Antigen retrieval was carried out in $0.01 \mathrm{~mol} / \mathrm{L}-1$ sodium citrate buffer (pH6) in a microwave for $20 \mathrm{~min}$; $10 \mathrm{~min}$ at full power $(750 \mathrm{~W})$ followed by $10 \mathrm{~min}$ at low power $(450 \mathrm{~W})$. Endogenous hydrogen peroxidase activity was then blocked in $0.3 \%$ hydrogen peroxide in methanol for $10 \mathrm{~min}$. Sections were incubated with the primary antibody diluted in antibody diluent (DAKO, Denmark) (1:100 for CD34 and D2-40) for $1 \mathrm{~h}$ at room temperature. Unbound primary antibody was washed with TBS prior to the addition of HRP-labeled polymer for $30 \mathrm{~min}$. Sections were then washed and immunohistochemical reactions were developed using 3, 3' diaminobenzidine (Liquid DAB+ Substrate Chromogen System, DAKO, K3468) for $7 \mathrm{~min}$.

Counterstained with haematoxylin was carried out for $3 \mathrm{~min}$ and rinsed off under running tap water. Sections were dehydrated in a series of ascending ethanol concentrations (30,50,70, 90 and $100 \%$ in water for $1 \mathrm{~min}$ at each concentration), fixed in xylene and mounted with DPX. Sections were left to dry overnight before viewing under the microscope. Tonsil sections were used as both positive and negative controls each time staining was conducted. The procedure as above was applied for positive controls. For negative controls, primary antibody was omitted.

\section{Microscopic analysis}

All microscopic analysis was carried out using light microscope (Olympus, Japan).

\section{Assessment of microvessel density and lymphatic vessel density}

Microvessel density was assessed by counting three hotspots with the highest number of vessels at 100x magnification. The mean value of the hotspots was used in the analysis. For lymphatic vessel density, the positively stained D240 vessels were counted manually across the tissue section. This ensure the accuracy of result as lymphatic vessels are present in much lower density that blood vessel density. For vessel density, both the peritumoral and intratumoral area were counted. Intratumoral was defined as the area within the tumor while peritumoral was defined as the peripheral area at 1 microscopic field of view $(\times 200)$ from the intratumoral area.

\section{Assessment of vascular invasion}

Figure 1 shows the examples of immunohistochemistry staining with D240 antibody. Lymphatic vessel invasion was identified as the presence of tumor cells within a D2-40 stained vessel. As CD34 can also stain a subset of lymphatic vessels, blood vessel invasion was defined when tumour cells were detected in CD34 positive but D240 negative vessels. The frequency of vascular invasion detected by H\&E staining was compared with that detected by IHC. False positive cases were recorded when $H \& E$ sections were positive for vascular invasion but negative in IHC. False negatives were recorded when vascular invasion was negative in the $H \& E$ sections but positive in IHC. $20 \%$ of the specimens were randomly chosen and analysed by second scorer blinded to the results to measure concordance between observers. 
Table 1 Clinicopathological characteristics of breast carcinoma patients and tumors

\begin{tabular}{|c|c|c|c|}
\hline Clinical criteria & Frequency, n (\%) & Clinical criteria & Frequency, n (\%) \\
\hline Age of diagnosis & & Recurrence & \\
\hline$\leq 40$ & $4(6.9)$ & No & $56(96.6)$ \\
\hline$\geq 40$ & $54(93.1)$ & Yes & $2(3.4)$ \\
\hline Ethnicity & & Estrogen Receptor & \\
\hline Malay & $54(93.1)$ & Positive & $32(55.2)$ \\
\hline Chinese & $4(6.9)$ & Negative & $26(44.8)$ \\
\hline Tumor Size (mm) & & Progesteron Receptor & \\
\hline$<30$ & $11(19.0)$ & Positive & $28(48.3)$ \\
\hline $30 \leq x<60$ & $27(46.6)$ & Negative & $30(51.7)$ \\
\hline$>60$ & $17(29.3)$ & & \\
\hline No data & $3(5.1)$ & & \\
\hline Tumor Grading & & Lymph nodes involvement & \\
\hline Grade I & $10(17.2)$ & 1 & $19(32.8)$ \\
\hline Grade II & $27(46.6)$ & $2-4$ & $6(10.3)$ \\
\hline Grade III & $16(27.6)$ & $>4$ & $16(27.6)$ \\
\hline Not determined & $5(8.6)$ & Not involved & $17(29.3)$ \\
\hline Distant Metastasis & & Lymphovascular Invasion (H\&E) & \\
\hline Yes & $15(25.9)$ & Positive & $26(44.8)$ \\
\hline No & $37(63.8)$ & Negative & $28(48.3)$ \\
\hline Not determined & $6(10.3)$ & Not determined & $4(6.9)$ \\
\hline \multicolumn{4}{|l|}{ Mastectomy } \\
\hline Full & $10(17.2)$ & & \\
\hline Partial & $48(82.8)$ & & \\
\hline
\end{tabular}

\section{Statistical analysis}

Vessels density was classified into high and low category based on the median value. Vessel invasion was divided into negative and positive groups and used as a basis to determine association between all parameters. The relationship between vascular invasion, vessels density and clinical criteria were assessed using chi square test (Fisher exact if the cell count was less than 5). Multivariate analyses were carried out using binomial logistic regression and multiple regression analysis based on the type of variables. Some tumors were not scored because of missing tissue and lack of peritumoral area. $\mathrm{P}$ value of less than 0.05 determined significant relationship. Variations between observers were measured using kappa score. Statistical analysis was carried out using IBM SPSS Statistic 24.

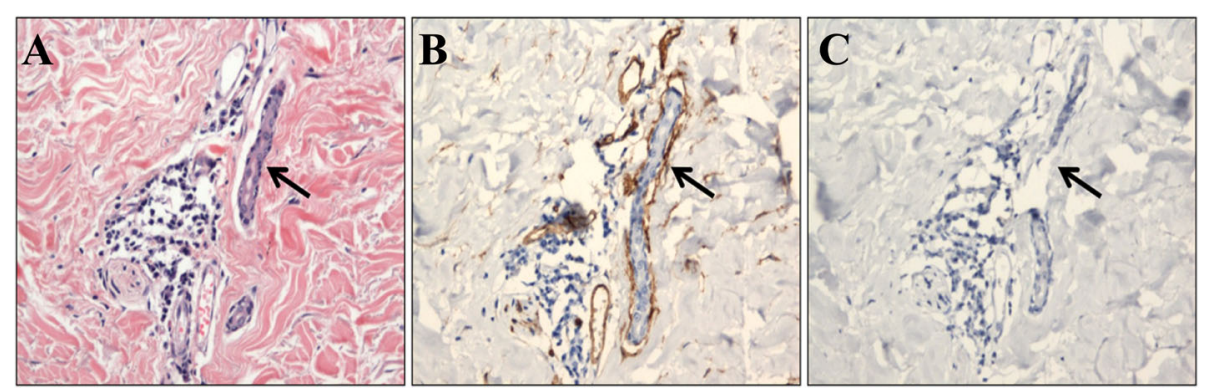

Fig. 1 Example of true positive lymphatic vessel invasion determined in D2-40 stained tissue. H\&E stained tissue scored as positive lymphovascular invasion (a). At the same field of microscopic field view (200x magnification), lymphatic vessel invasion was clearly observed in D2-40 stained tissue (b). In CD34 stained tissue (c), blood vessel invasion was scored as negative 


\section{Results}

\section{Distribution of lymphatic and blood vessels}

Microvessel density ranged from 5 to 26 vessels with a median of 10.3 vessels. Peritumoral lymphatic vessel density and intratumoral lymphatic vessel density showed a median of $0.052 / \mathrm{mm}^{2}$ and $0.078 / \mathrm{mm}^{2}$ respectively.

\section{Frequency of lymphatic and blood vessels invasion}

25.7\% ( $n=15)$ showed no vascular invasion on both intratumoral and peritumoral area. Of the 43 samples with vascular invasion, $69.8 \%(n=30)$ showed lymphatic vessel invasion. Of the LVI positive specimens, $33.3 \%(n=10), 26.7 \%(n=8)$ and $40.0 \%(n=12)$ were intratumoral invasion, peritumoral invasion and both intratumoral and peritumoral invasion respectively.

In comparison to IHC staining, only $55.2 \% \quad(n=32)$ showed invasion positive in $\mathrm{H} \& \mathrm{E}$ stained slides. When compared with all cases, 7 cases were false positive while 8 cases showed false negative results. Figure 1 shows the example of positive D2-40 staining which was scored as vascular invasion negative in H\&E section. Figure 2 shows the determination of lymphatic vessel invasion positive which was scored as invasion positive in $\mathrm{H} \& \mathrm{E}$ staining. The kappa scores of invasion using immunohistochemical markers between observers were 0.87 and 0.88 for CD34 and D240 respectively. The kappa score of $\mathrm{H} \& \mathrm{E}$ staining between observers was 0.61 .

\section{Association of lymphatic and blood vessel density with clinical criteria}

Table 2 shows the association of lymphatic vessel density with clinical criteria. Peritumoral and total lymphatic vessel density was significantly associated with age $(p=0.020$ and 0.017 respectively) Total lymphatic vessel density was also associated with grade $(p=0.018)$. Interestingly, peritumoral lymphatic vessel density was significantly associated with distant metastasis $(p=0.049)$. Blood vessel density was not associated with all clinical criterias.
However, in multivariate analysis none of these variables retain their significant association (peritumoral lymphatic vessel density: age $p=0.820$, distant metastasis $p=0.291$ and total lymphatic vessel density: grade $p=0.728$; age $p=0.916$ ).

\section{Association of lymphatic and blood vessel invasion with clinical criteria}

Table 3 shows the association of lymphatic vessel invasion with clinical criteria. Peritumoral lymphatic invasion was significantly associated with age $(p=0.012)$ and distant metastasis $(p=0.05)$. Blood vessel invasion was not significantly associated with all clinical criterias. In multivariate analysis, only age retain the significant association with peritumoral lymphatic vessel invasion $(p=0.001)$.

\section{Discussion}

The aim of this study was to compare the incidence of lymphatic invasion and blood invasion in breast carcinoma cohort between conventional H\&E staining and immunohistochemical staining. It also aimed to investigate the association of lymphatic/blood vessel density and invasion with adverse clinical criteria.

Previous studies showed that lymphatic invasion and blood vessel invasion cannot be distinguished when $H \& E$ staining was used in tissue biopsy $[8,9]$. The presence of lymphatic and blood endothelial cells specific markers, D2-40 and CD34, respectively made the identification of these vessels possible. It is important to know which type of invasion occurs in individual patients, which has been shown in this study because blood and lymphatic vessel invasion have different prognostic outcomes. In addition, personalized therapy could be designed to cater the needs of every patients based on their invasion routes. Furthermore, this study demonstrated that the concordance score between observers with the usage of immunohistochemical markers is excellent compared to the lower score when reporting

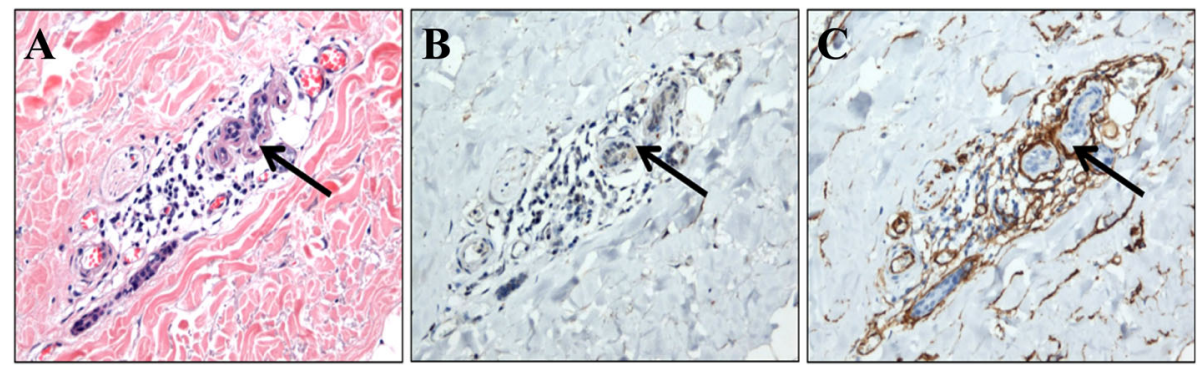

Fig. 2 H\&E (a), D2-40 (b) and CD-34 (c) staining of consecutive tissue section. This sample was scored as lymphovascular invasion negative by H\&E. However, CD34 staining (c) clearly showed the presence of tumor cells within CD34 stained vessel and was scored as blood vessel invasion positive. Lymphatic invasion was scored as negative (b). Noted that IHC was able to distinguih between lymphatic and blood vessel invasion. (200× magnification) 
Table 2 Association of lymphatic vessel density with clinical criteria

\begin{tabular}{|c|c|c|c|c|c|c|c|c|c|c|}
\hline & & Total LVD & & & Intra-tum & ral LVD & & Peri-tumo & al LVD & \\
\hline & & Low & High & $p$-value & Low & High & $p$-value & Low & High & $p$-value \\
\hline Age & $<40$ & $0(0.0)$ & $4(13.3)$ & 0.020 & $2(7.4)$ & $2(6.9)$ & 0.941 & $0(0.0)$ & $4(13.8)$ & 0.017 \\
\hline & $\geq 40$ & $27(100.0)$ & $26(86.7)$ & & 25 (92.6) & $27(93.1)$ & & $28(100.0)$ & $2(86.2)$ & \\
\hline Tumor size & $<30$ & $2(7.7)$ & $9(31.0)$ & 0.080 & $3(11.1)$ & $8(28.6)$ & 0.255 & $3(11.1)$ & $8(28.6)$ & 0.255 \\
\hline & $30 \leq x<60$ & 15 (57.7) & $12(41.4)$ & & 15 (55.6) & $12(42.9)$ & & 15 (55.6) & $12(42.9)$ & \\
\hline & $60 \geq$ & $9(34.6)$ & $8(27.6)$ & & $9(33.3)$ & $8(28.6)$ & & $9(33.3)$ & $8(28.6)$ & \\
\hline Grade & 1 & $2(7.7)$ & $8(26.7)$ & 0.018 & $1(3.7)$ & $9(31.0)$ & 0.030 & $13(11.1)$ & $7(24.1)$ & 0.079 \\
\hline & $\|$ & $13(50.0)$ & $14(46.7)$ & & 15 (55.6) & $12(41.4)$ & & $14(51.9)$ & $13(44.8)$ & \\
\hline & III & $11(42.3)$ & $5(16.7)$ & & $10(37.0)$ & $6(20.7)$ & & $10(37.0)$ & $6(20.7)$ & \\
\hline & No grade & 0 & $3(10.0)$ & & $1(3.7)$ & $2(6.9)$ & & 0 & $3(10.3)$ & \\
\hline Distant metastasis & Yes & $7(25.9)$ & $8(27.6)$ & 0.989 & $8(29.6)$ & $7(23.3)$ & 0.065 & $7(25.0)$ & $8(27.6)$ & 0.049 \\
\hline & No & $18(66.7)$ & $19(65.5)$ & & 19 (70.4) & 19 (63.3) & & $21(75.0)$ & 17 (58.6) & \\
\hline & Not determine & $2(7.4)$ & $2(6.9)$ & & 0 & $4(13.3)$ & & 0 & $4(13.8)$ & \\
\hline Recurrence & No & $26(96.3)$ & $29(96.7)$ & 0.940 & $27(100)$ & 27 (93.1) & 0.1 & $27(96.4)$ & $28(96.6)$ & 0.980 \\
\hline & Yes & $1(3.7)$ & $1(3.3)$ & & 0 & $2(6.9)$ & & $1(3.6)$ & $1(3.4)$ & \\
\hline ER & Positive & $16(61.5)$ & $16(55.2)$ & 0.632 & $16(61.5)$ & 15 (53.6) & 0.554 & $17(63.0)$ & $15(53.6)$ & 0.480 \\
\hline & Negative & $10(38.5)$ & $13(44.8)$ & & $10(38.5)$ & $13(46.4)$ & & $10(37.0)$ & $13(46.4)$ & \\
\hline$P R$ & Positive & $15(55.6)$ & $13(44.8)$ & 0.422 & $13(48.1)$ & $14(50.0)$ & 0.891 & $16(57.1)$ & $12(42.9)$ & 0.284 \\
\hline & Negative & $12(44.4)$ & $16(55.2)$ & & $14(51.9)$ & $14(50.0)$ & & $12(42.9)$ & $16(57.1)$ & \\
\hline Lymph nodes involvement & 0 & $8(29.6)$ & $11(37.9)$ & 0.374 & $8(30.8)$ & $11(37.9)$ & 0.678 & $7(25.0)$ & $12(42.9)$ & 0.266 \\
\hline & 1 & $3(11.1)$ & $3(10.3)$ & & $3(11.5)$ & $3(10.3)$ & & $4(14.3)$ & $2(7.1)$ & \\
\hline & 2 to 4 & $6(22.2)$ & $10(34.5)$ & & $6(23.1)$ & $10(31.0)$ & & $7(25)$ & $9(32.1)$ & \\
\hline & $>4$ & $10(37.0)$ & $5(17.2)$ & & $9(34.6)$ & $6(20.7)$ & & $10(35.7)$ & $5(17.9)$ & \\
\hline
\end{tabular}

*Bold datas refer to statistically significant association $(p<0.05)$

vascular invasion of $\mathrm{H} \& \mathrm{E}$ stained tissue section [5]. These showed that immunohistochemical markers could be used in clinical setting with patients samples to minimized the rate of error.

In this study, it was shown that although the frequency of blood vessel density was higher compared to lymphatic vessel density, the incidence of lymphatic vessel invasion exceeded that of blood vessel invasion. Clearly, breast carcinoma preferentially used lymphatic vessels as a route of metastasis. Similar data were also reported by previous study showing that lymphatic vessel invasion incidences exceeded of that blood vessel invasions which were 35 and 16\% respectively. Mohammed and colleagues also showed significant invasions of the lymphatics (97\%) compared to the blood vessels (3\%). These results and our results indicated clearly that breast carcinoma preferentially used lymphatic vessels as a route of metastasis. It was not known what drive tumor cells to invade lymphatic vessels when there are higher number of blood vessel in the tumoral area. One explanation is the anatomical structure of the lymphatic vessel itself which would not offer a significant barrier for the entry of tumor cells [10]. The lack of basement membrane and supporting structure in the lymphatic vasculature may ease the intravasation of tumor cells into the lymphatic circulation. In contrary, lymphatic endothelium might also play an active role in tumor cells recruitment. They may secrete chemokines that attracted the tumor cells to the lymphatic capillaries. Chemokines receptor-ligand relationships are important to regulate leukocytes trafficking and this relationship was hypothesized to be exploited by cancer cells to modulate entry into the lymphatic circulation [11, 12].

The presence of intratumoral lymphatic vessel invasion has been debatable as it was thought that lymphatic vessel could not penetrate the high pressure environment inside the tumor mass. With the discovery of new molecular markers and better imaging systems, the presence of intratumoral lymphatics has been reported [13]. In this study, we reported the presence of intratumoral lymphatic vessel invasion however no significant association was observed with any clinical criteria under study. Previous study has demonstrated the association between intratumoral lymphatic invasion with markers of aggressiveness [14] which was 
Table 3 Association of lymphatic vessel invasion with clinical criteria

\begin{tabular}{|c|c|c|c|c|c|c|c|c|c|c|}
\hline & & \multicolumn{3}{|c|}{ Intra-tumoral LVI } & \multicolumn{3}{|c|}{ Peri-tumoral LVI } & \multicolumn{3}{|l|}{ Total LVI } \\
\hline & & Positive & Negative & $p$-value & Positive & Negative & $p$-value & Positive & Negative & $p$-value \\
\hline \multirow[t]{2}{*}{ Age } & $<40$ & $2(7.1)$ & $2(7.1)$ & 1.0 & $4(14.8)$ & $0(0)$ & 0.012 & $4(10.5)$ & $0(0)$ & 0.065 \\
\hline & $\geq 40$ & $26(92.9)$ & $26(92.9)$ & & $23(85.2)$ & $30(100)$ & & 34 (89.5) & $19(100)$ & \\
\hline \multirow[t]{3}{*}{ Tumor size } & $<30$ & $7(25)$ & $4(14.8)$ & 0.548 & $4(14.8)$ & $7(25)$ & 0.636 & $9(23.7)$ & $2(11.8)$ & 0.497 \\
\hline & $30 \leq x<60$ & $12(42.9)$ & $15(55.6)$ & & $14(51.9)$ & $13(46.4)$ & & $17(44.7)$ & $10(58.8)$ & \\
\hline & $60 \geq$ & $9(32.1)$ & $8(29.6)$ & & $9(33.3)$ & $8(28.6)$ & & $12(31.6)$ & $5(29.4)$ & \\
\hline \multirow[t]{4}{*}{ Grade } & I & $3(10.7)$ & $7(25.0)$ & 0.387 & $5(18.5)$ & $5(17.2)$ & 0.197 & $5(13.2)$ & $5(27.8)$ & 0.607 \\
\hline & ॥ & $14(50.0)$ & $13(46.4)$ & & 15 (55.6) & $12(41.4)$ & & $19(50)$ & $8(44.4)$ & \\
\hline & III & $10(35.7)$ & $6(21.4)$ & & $7(25.9)$ & $9(31.0)$ & & $12(31.6)$ & $4(22.2)$ & \\
\hline & No grade & $1(3.33)$ & $2(7.1)$ & & 0 & $3(10.3)$ & & $2(5.3)$ & $1(5.6)$ & \\
\hline \multirow[t]{3}{*}{ Distant metastasis } & Yes & $9(32.1)$ & $6(21.4)$ & 0.432 & $7(25.9)$ & $8(26.7)$ & 0.05 & $11(28.9)$ & $4(21.1$ & 0.607 \\
\hline & No & $18(64.3)$ & $19(67.9)$ & & $20(74.1)$ & $18(60.0)$ & & $25(65.8)$ & $13(68.4)$ & \\
\hline & Not determine & $1(3.6)$ & $3(10.7)$ & & 0 & $4(13.3)$ & & $2(5.3)$ & $2(10.5)$ & \\
\hline \multirow[t]{2}{*}{ Recurrence } & No & $26(92.9)$ & $28(100)$ & 0.92 & $27(100)$ & $28(93.3)$ & 0.105 & $36(94.7)$ & $19(100)$ & 0.198 \\
\hline & Yes & $2(7.1)$ & 0 & & 0 & $2(6.7)$ & & $2(5.3)$ & 0 & \\
\hline \multirow[t]{2}{*}{ ER } & Positive & $16(59.3)$ & 15 (55.6) & 0.632 & $15(57.7)$ & $17(58.6)$ & 0.944 & $21(56.8)$ & $11(61.1)$ & 0.738 \\
\hline & Negative & $11(40.7)$ & $12(44.4)$ & & $11(42.3)$ & $2(41.4)$ & & $16(43.2)$ & $7(38.9)$ & \\
\hline \multirow[t]{2}{*}{$P R$} & Positive & $13(46.4)$ & $14(51.9)$ & 0.687 & $13(48.1)$ & $15(51.7)$ & 0.789 & $17(44.7)$ & $11(61.1)$ & 0.251 \\
\hline & Negative & $15(53.6)$ & $13(48.1)$ & & $14(51.9)$ & $14(48.3)$ & & $21(55.3)$ & $7(38.9)$ & \\
\hline \multirow[t]{4}{*}{ Lymph nodes involvement } & 0 & $10(37.0)$ & $9(32.1)$ & 0.983 & $7(25.9)$ & $12(41.4)$ & 0.088 & $13(35.1)$ & $6(31.6)$ & 0.763 \\
\hline & 1 & $3(11.1)$ & $3(10.7)$ & & $1(3.7)$ & $5(17.2)$ & & $3(8.1)$ & $3(15.8)$ & \\
\hline & 2 to 4 & $7(25.9)$ & $8(28.6)$ & & $11(40.7)$ & $5(17.2)$ & & $10(27)$ & $6(31.6)$ & \\
\hline & $>4$ & $7(25.9)$ & $8(28.6)$ & & $8(29.6)$ & $7(24.1)$ & & $11(29.7)$ & $4(21.1)$ & \\
\hline
\end{tabular}

*Bold datas refer to statistically significant association $(p<0.05)$

not observed in this cohort perhaps due to the number of samples used.

The clear association observed between peritumoral lymphatic vessel density and invasion with distant metastasis indicates that peritumoral lymphatic vessels have important role in breast carcinoma metastasis to secondary organs. Distant metastasis is a major factor that lead to poor prognosis of cancer patients. Statistics shows that $90 \%$ of cancer-related death occurs as a result of metastasis [15]. The highly significant association between these two variables may indicate that targeted therapy directed to peritumoral density could be designed to reduce metastatic dissemination of cancer cells especially in breast carcinoma patients.

\section{Conclusions}

In conclusion, although blood vessel density in breast carcinoma patients is higher compared to lymphatic vessel density, vascular invasion in breast carcinoma is predominantly lymphatic vessel invasion. The fact that peritumoral lymphatic vessel invasion was strongly correlated with distant metastasis shows that it is a strong predictor of breast carcinoma outcome. This study should be repeated in larger cohort with relapse-free survival and overall survival data. We strongly reccommended that IHC markers be used alongside H\&E staining to improve the detection rate and false interpretation of tissue samples.

\section{Abbreviations}

BVI: Blood vessel invasion; FFPE: Formalin fixed paraffin embedded; H\&E: Hematoxylin and eosin; LVI: Lymphatic vessel invasion

\section{Acknowledgements}

The authors thank Faezahtul Arbaeyah Hussain for technical support.

\section{Funding}

This work was supported by Short Term Research Grant, Universiti Sains Malaysia (grant number: 304/PPSK/61312137).

\section{Availability of data and materials}

The datasets used and analyzed during the current study are not publicly available to preserve patients' privacy but are available form the corresponding author on reasonable request.

\section{Authors' contributions}

NFN was involved in the acquisition and analysis of data and drafting manuscript. CCY collected the samples and performed staining. SS analysed the data, drafted and revised the manuscript. All authors read and approved the final manuscript. 


\section{Competing interests}

The authors declare that they have no competing interests.

\section{Consent for publication}

Not applicable.

\section{Ethics approval and consent to participate}

Ethical approval for this study was granted by the Human Research Ethics Committee, Universiti Sains Malaysia (304/PPSK/61312137). The need for individual informed consent was waived because of the use of archival materials.

Received: 24 February 2016 Accepted: 30 January 2017

Published online: 10 February 2017

\section{References}

1. Jemal A, Siegel R, Ward E, Hao Y, Xu J, Murray T, Thun MJ. Cancer Statistics, 2008. CA: A Cancer Journal for Clinicians CA: Cancer J Clin. 2008;58:26.

2. Dobi $A$, et al. Breast cancer under 40 years of age: increasing number and worse prognosis. Pathol Oncol Res. 2011;17(2):425-8.

3. Storr SJ, et al. Objective assessment of blood and lymphatic vessel invasion and association with macrophage infiltration in cutaneous melanoma. Mod Pathol. 2011;25:493-504.

4. Longatto-Filho A, et al. Lymphatic vessel density and epithelial D2-40 immunoreactivity in pre-invasive and invasive lesions of the uterine cervix. 2007. p. 45-51.

5. Mohammed R, et al. Improved methods of detection of lymphovascular invasion demonstrate that it is the predominant method of vascular invasion in breast cancer and has important clinical consequences. Am J Surg Pathol. 2007;31:9.

6. Mohammed RAA, et al. Objective assessment of lymphatic and blood vascular invasion in lymph node-negative breast carcinoma: findings from a large case series with long-term follow-up. J Pathol. 2011;223(3):358-65.

7. Doeden $\mathrm{K}$, et al. Lymphatic invasion in cutaneous melanoma is associated with sentinel lymph node metastasis. J Cutan Pathol. 2009;36(7):772-80.

8. Essner R. Sentinel lymph node biopsy and melanoma biology. 2006. p. 2320s-5s.

9. El-Gohary YM, et al. Prognostic significance of intratumoral and peritumoral lymphatic density and blood vessel density in invasive breast carcinomas. 2008. p. 578-86.

10. Witte $M$, et al. Structure function relationships in the lymphatic system and implications for cancer biology. Cancer Metastasis Rev. 2006;25(2):159-84.

11. Kawai $Y$, et al. Chemokine CCL2 facilitates ICAM-1-mediated interactions of cancer cells and lymphatic endothelial cells in sentinel lymph nodes. Cancer Sci. 2009;100(3):419-28.

12. Johnson LA, Jackson DG. Cell traffic and the lymphatic endothelium. Ann N Y Acad Sci. 2008;1131(1):119-33.

13. Ji RC, Eshita $Y$, Kato $S$. Investigation of intratumoural and peritumoural lymphatics expressed by podoplanin and LYVE-1 in the hybridoma-induced tumours. Int J Exp Pathol. 2007:88(4):257-70.

14. Straume $O$, Akslen LA. Lymphatic vessel density and prognosis in cutaneous melanoma. Br J Cancer. 2004;91(6):1224-5.

15. Parkin DM, et al. Fifty years of cancer incidence. Int J Cancer. 2010;127(12): 2918-27.

\section{Submit your next manuscript to BioMed Central and we will help you at every step:}

- We accept pre-submission inquiries

- Our selector tool helps you to find the most relevant journal

- We provide round the clock customer support

- Convenient online submission

- Thorough peer review

- Inclusion in PubMed and all major indexing services

- Maximum visibility for your research

Submit your manuscript at www.biomedcentral.com/submit 